\title{
Total oxidant/antioxidant status in sera of patients with thyroid cancers
}

\author{
Dong Wang ${ }^{1 *}$, Jia-Fu Feng ${ }^{2 *}$, Ping Zeng ${ }^{2}$, Yun-Hong Yang ${ }^{3}$, Jun Luo ${ }^{2}$ \\ and Yu-Wei Yang ${ }^{2}$
}

Departments of ${ }^{1}$ Oncology, ${ }^{2}$ Laboratory and ${ }^{3}$ Pathology, Mianyang Central Hospital, Mianyang, Sichuan Province, China

(Correspondence should be addressed to J-F Feng; Email: jiafufeng@yahoo.com.cn)

*(D Wang and J-F Feng contributed equally to this work)

\begin{abstract}
Oxidative stress is considered to be involved in the pathophysiology of all cancers. In order to evaluate the total oxidant/antioxidant status in patients with thyroid cancer and to investigate the relationship between oxidative stress parameters and serum thyroid profiles among thyroid cancer patients and various controls, we determined oxidative status including total antioxidant status (TAS) and total oxidant status (TOS) and calculation of oxidative stress index (OSI) in sera in 82 thyroid cancer patients, 56 benign thyroid disease patients, and 50 healthy controls. It was found that serum TAS levels were significantly lower in patients with thyroid cancer than in controls $(P<0.001$ ), while serum TOS levels and OSI values were significantly higher (both $P<0.001$ ) in the cancer patients. No significant correlations were observed between various oxidative stress markers and thyroid profiles in either the thyroid cancer patients or the controls. Receiver operating characteristic curve analysis demonstrated that OSI was the best indicator for distinguishing cancer patients from benign thyroid diseased or healthy controls, followed by TOS and TAS. Risk estimate statistics also indicated that TOS and/or OSI were good risk factors to discriminate patients with thyroid cancer from two controls. These findings suggested that oxidants are increased and antioxidants are decreased in patients with thyroid cancer. OSI may be a more useful oxidative stress biomarker than TAS and TOS for monitoring the clinical status of thyroid cancer patients.
\end{abstract}

Endocrine-Related Cancer (2011) 18 773-782

\section{Introduction}

Thyroid cancer is the most common endocrine malignancy and its incidence continues to rise yearly. Thyroid carcinoma, in most cases, presents clinically as a solitary nodule or as a dominant nodule within a multinodular thyroid gland. The challenge to clinicians is to discriminate between the minority of thyroid nodules (5-15\%) that harbor malignancies and the majority of cases that can be managed conservatively (Boelaert 2009). Thus, it is very important to identify one or more markers to distinguish thyroid cancer from different types of thyroid disease.

Cellular oxidative damage is a well-established general mechanism of cell and tissue injury that is primarily caused by free radicals and reactive oxygen species (ROS). Low levels of ROS are indispensable in many biochemical processes (Valko et al. 2007); however, overproduction and/or inadequate removal of ROS can result in oxidative stress, which is characterized as an imbalance between the formation of active oxygen metabolites and the rate at which they are scavenged by enzymatic and non-enzymatic antioxidants. Oxidative stress can participate in the pathogenesis and complications of many diseases (including cancer; Uttara et al. 2009).

Thyroid hormones regulate oxidative metabolism and thus play an important role in free radical production (Erdamar et al. 2008). Thyroid hormones regulate the synthesis and degradation of enzymes, such as superoxide dismutase (SOD), catalase, glutathione peroxidase (GPx), and glutathione reductase, and non-enzymatic antioxidants, such as vitamin $\mathrm{E}$ and $\mathrm{C}$, glutathione, uric 
acid, ferritin, transferrin, and ceruloplasmin. Undoubtedly, the changes in these enzymes and non-enzymatic substances affect the redox balance in the body and, in turn, enzymatic feedback regulates thyroid function. One of the major effects of thyroid hormones is to increase mitochondrial respiration, which results in upregulation of ROS, leading to oxidative damage to membrane lipids (Erdamar et al. 2008).

In recent years, interest has grown in studying the role played by oxidative stress in thyroid carcinogenesis by investigating one or more of the antioxidant markers, including SOD (Laatikainen et al. 2010, Lassoued et al. 2010, Erdamar et al. 2010), catalase (Lassoued et al. 2010), GPx (Lassoued et al. 2010, Erdamar et al. 2010), vitamin E (Alkhenizan and Hafez 2007), vitamin C (Liu et al. 2010), glutathione (Du et al. 2009), and/or uric acid (Abiaka et al. 2001). However, the measurement of different antioxidant molecules separately was not only impractical but also held no clinical significance. Because the effects of antioxidants can be additive and measuring individual antioxidants separately is time consuming and labor intensive, a measurement of the combined activities of all antioxidants or the total antioxidant status (TAS) is often used to estimate the overall antioxidative status (Erel 2004). Likewise, total oxidant status (TOS) is measured to determine a patient's overall oxidation state (Erel 2005). Furthermore, the oxidative stress index (OSI), which is calculated as the ratio of TOS to TAS, may be a more accurate index of oxidative stress in the body because it is a comprehensive measurement of TAS and TOS.

Oxidative stress is common in the thyroid tissue during utilization of $\mathrm{H}_{2} \mathrm{O}_{2}$ for thyroxine $\left(\mathrm{T}_{4}\right)$ synthesis, the inflammation produces ROS, and when tumor has active proliferation (Akinci et al. 2008). Therefore, oxidative stress has been reported to be consistently associated with hyperthyroidism or hypothyroidism (Erdamar et al. 2008). However, to date, our understanding of the roles of the oxidative/antioxidative systems in the carcinogenesis of thyroid cancer and their relationships to thyroid profiles is limited. To address this, we evaluated the levels of TAS, TOS, and OSI in thyroid cancer patients, benign thyroid disease patients, and healthy controls and analyzed the relationship among these oxidative stress parameters, thyroid profiles, and thyroid cancer.

\section{Materials and methods}

\section{Subjects}

From March 2005 to December 2010, 82 patients with thyroid cancer (35 males and 47 females) were involved in this study. All patients were newly diagnosed as having thyroid cancer based on clinical laboratory investigations confirmed by fine needle aspiration. The mean age of the patients was 33.4 \pm 7.9 years with a range of 12-53 years. Of these 82 cancer patients (cancer group), there were 53 papillary thyroid cancer (PTC) patients, 18 follicular thyroid cancer patients, six anaplastic differentiated thyroid cancer (ATC) patients, and five medullary thyroid cancer patients. In addition, there were 56 benign thyroid disease patients (22 males and 34 females; age range 15-62 years) that served as disease controls, including 36 patients with hyperthyroidism due to Graves' disease (hyperthyroidism group) and 20 patients with hypothyroidism due to Hashimoto's thyroiditis (hypothyroidism group). These patients were also newly diagnosed cases of clinical dysthyroidism.

Age-matched subjects from the same region $(n=50$; 19 males and 31 females; age range 17-56 years) were used as healthy controls (healthy group (HG)). HG blood samples were measured in parallel to obtain normal base line values.

The study protocol was approved by the Medical Ethics Committee of Mianyang Central Hospital, Sichuan province, China, and written informed consent was obtained from all legal guardians of tested participants.

\section{Blood samples}

After overnight fasting, blood was drawn by venipuncture at the antecubital vein from the subjects between 0730 and $1000 \mathrm{~h}$. Approximately $5 \mathrm{ml}$ blood was collected into a BD Vacutainer SST II ADVANCE tube (Becton Dickinson, Rutherford, NJ, USA) for analysis of oxidative stress markers and thyroid profiles. After $1 \mathrm{~h}$, blood samples were centrifuged at $700 \mathrm{~g}$ for $15 \mathrm{~min}$, and sera samples were collected and stored at $-30{ }^{\circ} \mathrm{C}$ until analysis within $48 \mathrm{~h}$.

\section{Thyroid profiles}

Thyroid profiles were assessed by estimation of serum-free triiodothyronine $\left(\mathrm{FT}_{3}\right)$, free $\mathrm{T}_{4}\left(\mathrm{FT}_{4}\right)$, and thyrotropic-stimulating hormone (TSH), which were assayed by a chemiluminescent assay method using a LIAISON analyzer with original kits obtained from DiaSorin (DiaSorin S.p.A, Saluggia, Italy). The $\mathrm{FT}_{3}$ to $\mathrm{FT}_{4}$ ratio $\left(\mathrm{FT}_{3} / \mathrm{FT}_{4}\right)$ was also calculated. 


\section{TAS determination}

TAS was measured colorimetrically using the TAS kit (Eurobiomed Randox, London, UK). This assay relies on the ability of antioxidants in the sample to inhibit the formation of $\mathrm{ABTS}^{+}$from oxidation of ABTS (2.2'-azino-di-(3-ethylbenz-thiazoline sulfonate)) by metmyoglobin (a peroxidase). An antioxidant of known concentration $(1.65 \mathrm{mmol} / \mathrm{l})$ was used as a standard for the calculation of antioxidant levels in the samples. Values of TAS were expressed as mmol Trolox equivalent/l (mmol Trolox equiv./l).

\section{TOS determination}

Serum TOS was measured using Erel's TOS method (Harma et al. 2005), which is based on the oxidation of ferrous ion to ferric ion in the presence of various oxidative species in acidic medium and the measurement of the ferric ion by xylenol orange. The test parameters were as follows: end-point measurement, serum $10 \mu \mathrm{l}, R_{1} 200 \mu \mathrm{l}, R_{2} 50 \mu \mathrm{l}, 10 \mathrm{~min}$ at $37^{\circ} \mathrm{C}$, read points 34 , primary wavelength $560 \mathrm{~nm}$, and secondary wavelength $800 \mathrm{~nm}$. The results were expressed in $\mu$ mol $\mathrm{H}_{2} \mathrm{O}_{2}$ equivalent/l ( $\mu$ mol $\mathrm{H}_{2} \mathrm{O}_{2}$ equiv./l).

\section{Oxidative stress index}

The TOS to TAS ratio was regarded as the OSI (Aycicek et al. 2005, Harma et al. 2005) and was calculated as follows: OSI (arbitrary unit $)=((\mathrm{TOS}$, $\mu \mathrm{mol} \mathrm{H}_{2} \mathrm{O}_{2}$ equiv./l)/(TAS, $\mu$ mol Trolox equiv./l) $\times$ 100) (Aycicek \& Erel 2007).

\section{Statistical analysis}

All data were expressed as the mean \pm s.D. and/or range (minimum-maximum). One-way ANOVA was used to determine the significance of biochemical parameters among the study groups. The least significant difference test was used for multiple comparisons to estimate the difference between the two study groups. Multiple linear regression analysis was performed to evaluate the associations between serum levels of oxidative stress markers and thyroid profiles. Receiver operating characteristic (ROC) curves, depicting the ability to discriminate between controls and malignant conditions, were plotted for the oxidative stress markers and reported the area under the curve (AUC) and its $95 \%$ confidence interval (CI). $P$ values $<0.05$ were considered statistically significant. All statistical analyses were carried out using the statistical package program version SSPS 17.0 (SPSS, Inc., Chicago, IL, USA).

\section{Results}

In this study, hyperthyroidism was defined as a basal serum TSH $<0.4 \mathrm{mIU} / \mathrm{l}, \mathrm{FT}_{4}>1.9 \mathrm{ng} / \mathrm{ml}$, and $\mathrm{FT}_{3}$ $>4.2 \mathrm{pg} / \mathrm{ml}$. Hypothyroidsm was defined as basal serum $\mathrm{TSH}>4.0 \mathrm{mIU} / \mathrm{l}, \mathrm{FT}_{4}<0.8 \mathrm{ng} / \mathrm{ml}$, and $\mathrm{FT}_{3}$ $<1.8 \mathrm{pg} / \mathrm{ml}$. Healthy controls included demonstrated a basal serum TSH $0.4-3.8 \mathrm{mIU} / 1, \mathrm{FT}_{4} 0.8-1.7 \mathrm{ng} / \mathrm{ml}$, and $\mathrm{FT}_{3} 2.2-4.2 \mathrm{pg} / \mathrm{ml}$.

The results are expressed as mean \pm s.D. with minimum and maximum values shown in Table 1. Samples of various concentrations were assayed to estimate repeatability and reproducibility of each

Table 1 Serum levels of oxidative stress markers and thyroid profiles in study groups (above: mean \pm s.D., below: min.-max.)

\begin{tabular}{lllllc}
\hline Items & CG (82) & HpeG (36) & HpoG (20) & HG (50) & Statistic values \\
\hline TAS & $1.37 \pm 0.20^{*, \neq, \S}$ & $1.52 \pm 0.13^{\dagger}$ & $1.53 \pm 0.11^{\dagger}$ & $1.67 \pm 0.15$ & $F=32.951$ \\
& $1.00-1.70$ & $1.33-1.77$ & $1.32-1.69$ & $1.45-1.95$ & $P=0.000$ \\
TOS & $21.34 \pm 2.82^{*, \neq, \S}$ & $15.79 \pm 1.41^{\dagger}$ & $16.08 \pm 1.47^{\dagger}$ & $14.87 \pm 1.01$ & $F=129.850$ \\
& $16.23-26.55$ & $13.51-18.82$ & $13.35-18.08$ & $13.22-16.95$ & $P=0.000$ \\
OSI & $1.60 \pm 0.31^{*, \neq, \S}$ & $1.05 \pm 0.11^{\dagger}$ & $1.06 \pm 0.11^{\dagger}$ & $0.89 \pm 0.07$ & $F=129.069$ \\
& $1.01-2.33$ & $0.82-1.23$ & $0.91-1.28$ & $0.69-1.09$ & $P=0.000$ \\
TSH & $0.221 \pm 0.354^{*, \S}$ & $0.028 \pm 0.027^{*}$ & $20.665 \pm 4.054^{*}$ & $1.627 \pm 0.766$ & $F=1269.993$ \\
& $0.002-2.075$ & $0.004-0.088$ & $15.908-29.664$ & $0.677-3.050$ & $P=0.000$ \\
$\mathrm{FT}_{3}$ & $2.82 \pm 0.59^{*, \S}$ & $7.16 \pm 1.40^{*}$ & $1.42 \pm 0.37^{*}$ & $3.02 \pm 0.42$ & $F=351.354$ \\
& $1.52-4.19$ & $5.45-9.70$ & $0.95-1.95$ & $2.25-3.95$ & $P=0.000$ \\
$\mathrm{FT}_{4}$ & $1.10 \pm 0.31^{\ddagger}$ & $15.76 \pm 6.62^{*}$ & $0.53 \pm 0.16$ & $1.22 \pm 0.24$ & $F=249.572$ \\
& $0.53-1.69$ & $5.62-28.12$ & $0.30-0.82$ & $0.82-1.67$ & $P=0.000$ \\
$\mathrm{FT}_{3} / \mathrm{FT}_{4}$ & $2.80 \pm 1.05^{\ddagger}$ & $0.57 \pm 0.32^{*}$ & $2.98 \pm 1.39^{\dagger}$ & $2.56 \pm 0.53$ & $F=60.484$ \\
& $0.90-5.46$ & $0.21-1.50$ & $1.45-6.23$ & $1.42-3.80$ & $P=0.000$ \\
\hline
\end{tabular}

Comparison with $\mathrm{HG},{ }^{*} P<0.001,{ }^{\dagger} P<0.05$; comparison with $\mathrm{HpeG},{ }^{\ddagger} P<0.001$; comparison with $\mathrm{HpoG},{ }^{\S} P<0.001$. Least significant difference (LSD) test. TAS, TOS, and OSI units are expressed as mmol Trolox equivalent/l, $\mu \mathrm{mol}_{2} \mathrm{O}_{2}$ equivalent/l, and arbitrary unit respectively. CG, cancer group; HpeG, hyperthyroidism group; HpoG, hypothyroidism group; HG, healthy group. 
$D$ Wang, J-F Feng et al.: Oxidative stress and thyroid cancer

Table 2 Estimation of the repeatability and reproducibility of each assay

\begin{tabular}{|c|c|c|c|c|c|c|}
\hline & \multicolumn{3}{|c|}{ Intra-assay } & \multicolumn{3}{|c|}{ Day-to-day } \\
\hline & Mean & $n$ & CVi (\%) & Mean & $n$ & CVd (\%) \\
\hline \multirow[t]{3}{*}{ TAS (mmol Trolox equiv./l) } & 0.92 & 20 & 1.75 & 0.93 & 20 & 3.13 \\
\hline & 1.26 & 20 & 1.22 & 1.30 & 20 & 3.46 \\
\hline & 1.90 & 20 & 1.79 & 2.03 & 20 & 2.87 \\
\hline \multirow[t]{3}{*}{ TOS ( $\mu \mathrm{mol} \mathrm{H}_{2} \mathrm{O}_{2}$ equiv./I) } & 13.06 & 20 & 2.01 & 13.22 & 20 & 2.76 \\
\hline & 16.88 & 20 & 1.89 & 18.72 & 20 & 2.53 \\
\hline & 26.43 & 20 & 1.91 & 26.19 & 20 & 2.56 \\
\hline \multirow[t]{4}{*}{$\mathrm{FT}_{3}(\mathrm{pg} / \mathrm{ml})$} & 1.01 & 20 & 4.6 & 1.96 & 20 & 6.3 \\
\hline & 2.01 & 20 & 2.8 & 3.64 & 20 & 4.2 \\
\hline & 3.80 & 20 & 1.7 & 9.79 & 20 & 2.9 \\
\hline & 13.80 & 20 & 1.4 & 13.05 & 20 & 3.9 \\
\hline \multirow[t]{5}{*}{$\mathrm{FT}_{4}(\mathrm{ng} / \mathrm{dl})$} & 0.39 & 20 & 2.4 & 0.48 & 23 & 4.8 \\
\hline & 0.90 & 20 & 1.6 & 0.90 & 31 & 3.7 \\
\hline & 1.47 & 20 & 1.1 & 1.53 & 32 & 2.9 \\
\hline & 3.00 & 20 & 2.4 & 3.02 & 31 & 3.3 \\
\hline & 6.36 & 20 & 2.0 & 6.36 & 30 & 3.2 \\
\hline \multirow[t]{3}{*}{ TSH (mlU/l) } & 0.17 & 20 & 1.9 & 0.17 & 21 & 5.1 \\
\hline & 10.4 & 20 & 0.7 & 10.5 & 21 & 1.6 \\
\hline & 36.1 & 20 & 0.9 & 36.8 & 21 & 2.2 \\
\hline
\end{tabular}

assay. The intra-assay and inter-assay coefficients of variation $(\mathrm{CVi}, \mathrm{CVd})$ are presented in Table 2.

One-way ANOVA showed (Table 1) significant differences among the study groups in the levels of oxidative stress markers TAS $(F=16.958, P=0.000)$, TOS $(F=55.796, P=0.000)$, and OSI $(F=56.419$, $P=0.000)$, as well as thyroid profile $\mathrm{TSH}(F=$ 813.033, $P=0.000), \mathrm{FT}_{3}(F=86.239, P=0.000), \mathrm{FT}_{4}$ $(F=191.500, P=0.000)$, and $\mathrm{FT}_{3} / \mathrm{FT}_{4}(F=37.327$, $P=0.000)$. These results indicated increased oxidative stress in both thyroid disease patients and thyroid cancer patients. However, no statistically significant differences were found in serum TAS, TOS, and OSI values among carcinoma histological subtypes (Table 3).

Multiple linear regression analysis were performed with the TAS, TOS, or OSI as dependent variables and using $\mathrm{TSH}, \mathrm{FT}_{3}, \mathrm{FT}_{4}$, and $\mathrm{FT}_{3} / \mathrm{FT}_{4}$ as independent variables. The results showed no significant correlations between various oxidative stress markers and thyroid profiles for either the thyroid cancer patients or the controls (Table 4).

ROC curves for the abilities of TAS, TOS, and OSI to distinguish between benign and malignant diagnoses are plotted in Fig. 1A-D respectively. Based on the results from the ROC curve analysis, the optimal cutoff

Table 3 Serum levels of oxidative stress markers in carcinoma histological subtypes

\begin{tabular}{lllllll}
\hline Items & Cancer $(n)$ & Mean & s.D. & Min. & Max. & Statistic values \\
\hline TAS & PTC (53) & 1.38 & 0.20 & 1.04 & 1.70 & $F=0.430, P=0.732$ \\
& FTC (18) & 1.35 & 0.22 & 1.00 & 1.63 & \\
& ATC (6) & 1.33 & 0.15 & 1.05 & 1.46 & \\
& MTC (5) & 1.29 & 0.25 & 1.04 & 1.56 & \\
TOS & TTC (82) & 1.37 & 0.20 & 1.00 & 1.70 & \\
& PTC (53) & 21.81 & 2.86 & 17.18 & 26.55 & \\
& FTC (18) & 20.80 & 2.60 & 16.23 & 23.96 & \\
& ATC (6) & 20.11 & 2.49 & 16.71 & 23.06 & \\
OSI & MTC (5) & 19.78 & 3.01 & 16.59 & 22.81 & \\
& TTC (82) & 21.34 & 2.82 & 16.23 & 26.55 & \\
& PTC (53) & 1.61 & 0.31 & 1.14 & 2.33 & \\
& FTC (18) & 1.59 & 0.37 & 1.01 & 2.33 & \\
& ATC (6) & 1.52 & 0.17 & 1.29 & 1.78 & \\
& MTC (5) & 1.56 & 0.20 & 1.31 & 1.86 & \\
\end{tabular}

PTC, papillary thyroid cancer; FTC, follicular thyroid cancer patients; ATC, anaplastic differentiated thyroid cancer; MTC, medullary thyroid cancer; TTC, total thyroid cancer. 
Table 4 Association between various oxidative stress markers and thyroid profiles by multiple linear regression analysis

\begin{tabular}{|c|c|c|c|c|c|c|c|}
\hline & $n$ & $\mathbf{T S H}(r, P)$ & $\mathbf{F T}_{\mathbf{3}}(r, P)$ & $\mathbf{F T}_{\mathbf{4}}(r, P)$ & $\mathbf{F T}_{\mathbf{3}} / \mathbf{F T}_{\mathbf{4}}(r, P)$ & $R$ & $F, P$ \\
\hline \multirow[t]{3}{*}{ TAS } & 50 & $-0.046,0.376$ & $0.034,0.408$ & $0.104,0.237$ & $-0.090,0.267$ & 0.142 & $0.230,0.920$ \\
\hline & 56 & $0.073,0.297$ & $0.011,0.469$ & $-0.011,0.467$ & $0.082,0.274$ & 0.196 & $0.511,0.827$ \\
\hline & 82 & $-0.031,0.391$ & $0.106,0.172$ & $-0.057,0.307$ & $0.081,0.234$ & 0.127 & $0.315,0.867$ \\
\hline \multirow[t]{3}{*}{ TOS } & 50 & $-0.004,0.488$ & $-0.043,0.385$ & $0.096,0.254$ & $-0.128,0.188$ & 0.149 & $0.255,0.905$ \\
\hline & 56 & $0.114,0.201$ & $-0.093,0.248$ & $0.069,0.307$ & $-0.015,0.456$ & 0.302 & $1.282,0.289$ \\
\hline & 82 & $-0.036,0.376$ & $0.132,0.118$ & $-0.170,0.063$ & $0.49,0.090$ & 0.275 & $1.578,0.189$ \\
\hline \multirow[t]{3}{*}{ OSI } & 50 & $0.069,0.316$ & $-0.054,0.356$ & $-0.034,0.408$ & $0.005,0.486$ & 0.104 & $0.122,0.974$ \\
\hline & 56 & $0.032,0.407$ & $-0.078,0.283$ & $0.071,0.301$ & $-0.084,0.270$ & 0.247 & $0.829,0.513$ \\
\hline & 82 & $-0.002,0.493$ & $-0.012,0.458$ & $-0.049,0.330$ & $0.008,0.471$ & 0.100 & $0.194,0.941$ \\
\hline
\end{tabular}

$r$, Pearson's correlation coefficient; $R$, multiple correlation coefficient.

points were estimated using the maximum values of the Youden index (YI). AUC, cutoff values, sensitivity, specificity, and YI are reported in Table 5. The results showed that the OSI was the best indicator for distinguishing cancer patients from disease controls and/or healthy controls, followed by TOS and TAS. Compared with disease controls, AUC (95\% CI) was 0.964 (0.933-0.994) for OSI, 0.929 (0.883-0.974) for TOS, and $0.702(0.599-0.805)$ for TAS. Compared with healthy controls, AUC $(95 \%$ CI) was 1.000 (1.000-1.000) for OSI, 0.996 (0.000-1.000) for TOS, and $0.878(0.803-0.954)$ for TAS. Between disease controls and healthy controls, the AUC $(95 \% \mathrm{CI})$ was 0.979 (0.960-0.998) for OSI, 0.957 (0.928-0.986) for TOS, and $0.776(0.697-0.855)$ for TAS. These data show that lower TAS and higher TOS levels (i.e. increased oxidative stress) may be related to thyroid cancer.
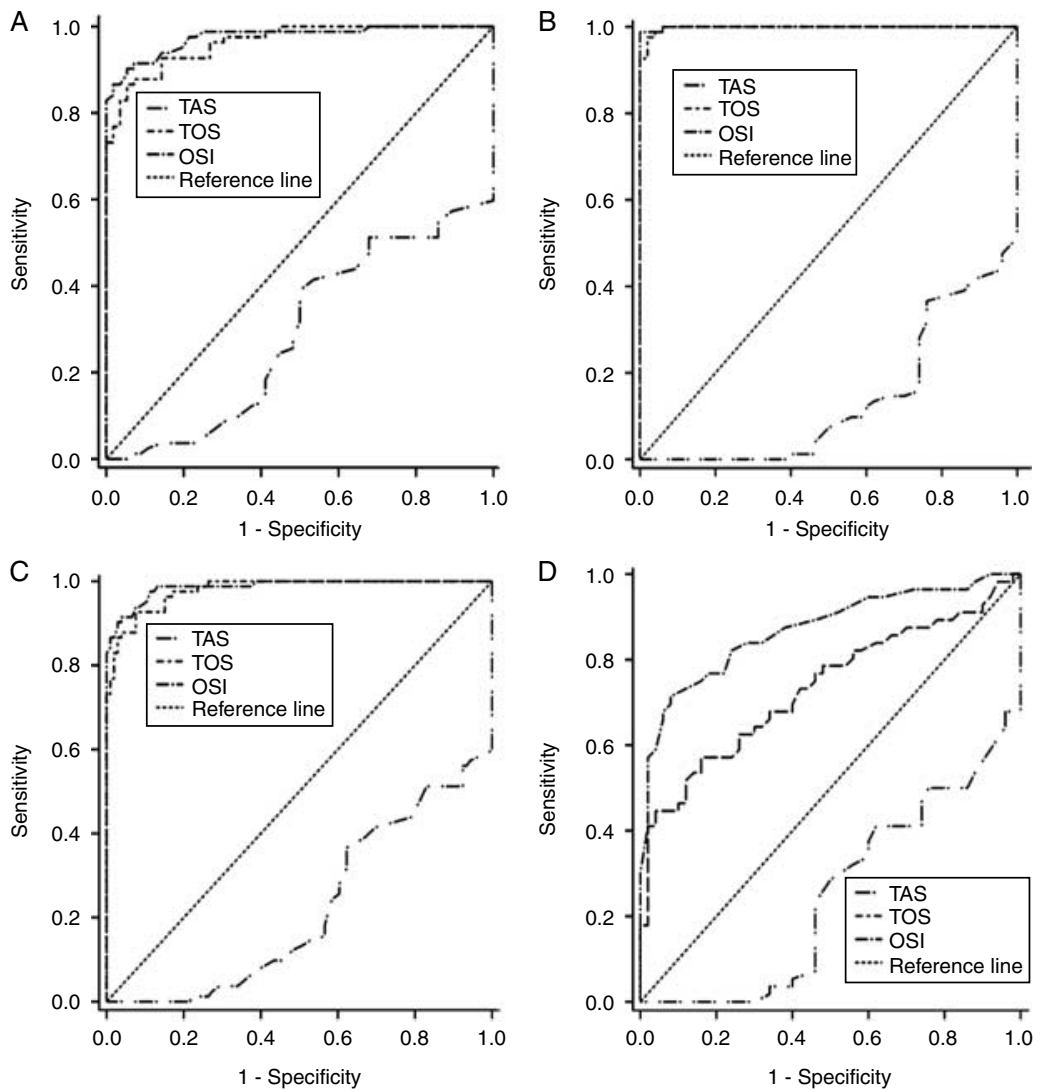

Figure 1 ROC curve analyses of the oxidative stress biomarkers. (A) (HpeG+HpoG) vs CG; (B) HG vs CG; (C) (HG + HpeG + HpoG) vs CG; (D) HG vs (HpeG+HpoG). The TAS curve is inverted because the results are decreased. 
Table 5 Performance of oxidative stress markers in predicting thyroid cancer

\begin{tabular}{|c|c|c|c|c|c|}
\hline & AUC $(95 \% \mathrm{Cl})$ & Cutoff & Se & Sp & YI \\
\hline \multicolumn{6}{|c|}{ (HpeG+HpoG) vs CG } \\
\hline TAS & $0.706(0.620-0.792)$ & 1.41 & 85.7 & 48.8 & 0.345 \\
\hline TOS & $0.963(0.938-0.989)$ & 17.17 & 92.7 & 85.7 & 0.784 \\
\hline OSI & $0.975(0.954-0.997)$ & 1.21 & 90.2 & 94.6 & 0.848 \\
\hline \multicolumn{6}{|c|}{ HG vs CG } \\
\hline TAS & $0.862(0.801-0.923)$ & 1.56 & 74.0 & 84.1 & 0.581 \\
\hline TOS & $0.998(0.000-1.000)$ & 16.54 & 97.6 & 98.0 & 0.956 \\
\hline OSI & $0.999(0.000-1.000)$ & 1.11 & 98.8 & 100.0 & 0.988 \\
\hline \multicolumn{6}{|c|}{$(\mathrm{HG}+\mathrm{HpeG}+\mathrm{HpoG})$ vs $\mathrm{CG}$} \\
\hline TAS & $0.780(0.715-0.845)$ & 1.41 & 92.5 & 48.8 & 0.413 \\
\hline TOS & $0.980(0.965-0.994)$ & 16.69 & 96.3 & 84.9 & 0.812 \\
\hline OSI & $0.987(0.974-0.999)$ & 1.19 & 91.5 & 96.2 & 0.877 \\
\hline \multicolumn{6}{|c|}{ HG vs (HpeG + HpoG) } \\
\hline TAS & $0.750(0.659-0.841)$ & 1.56 & 74.0 & 84.1 & 0.581 \\
\hline TOS & $0.734(0.639-0.829)$ & 16.83 & 57.1 & 84.0 & 0.411 \\
\hline OSI & $0.871(0.803-0.939)$ & 0.99 & 71.4 & 92.0 & 0.634 \\
\hline
\end{tabular}

TAS, TOS, and OSI units are expressed as mmol Trolox equiv./l, $\mu \mathrm{mol} \mathrm{H}_{2} \mathrm{O}_{2}$ equiv./l, and arbitrary unit respectively. Se, sensitivity; $\mathrm{Sp}$, specificity; YI, Youden index; CG, cancer group; HpeG, hyperthyroidism group; HpoG, hypothyroidism group; HG, healthy group.

Risk estimate statistics were calculated using the cutoff values obtained by ROC curve analysis. These results showed that the TOS and/or TOS were sufficient risk factors to discriminate thyroid cancer from benign thyroid diseases and/or healthy controls (Table 6).

\section{Discussion}

Aerobic organisms have developed complex antioxidant systems that can counteract ROS and free radicals. This system can prevent and/or reduce ROSand free radical-induced oxidative damage to tissues and cells (Wells et al. 2009) and is composed of different antioxidant substances, including the enzymes, nonenzymatic antioxidants, and an array of small molecules (Santi et al. 2010). These known and many unknown antioxidant substances compose the body's complex antioxidant system. To assess the antioxidant status in vivo, it is essential to measure the levels of overall antioxidants, including those known and unknown. In addition, the dynamic distribution of different antioxidants in various biological samples and their potential interactions make it difficult to measure each antioxidant separately, and such measurements are also unlikely to represent the overall antioxidant substances in the body. Similarly, to assess the oxidant status in vivo, it is essential to measure the levels of overall oxidants.

However, in previous studies concerning the role of antioxidants in thyroid cancer, most reports measured only one or several antioxidant substances separately
(Senthil \& Manoharan 2004, Akinci et al. 2008, Lin et al. 2009, Laatikainen et al. 2010, Young et al. 2010) such that the results did not reflect the total antioxidant levels in patients. In the current study, we recommended measuring the total oxidant/antioxidant status of an organism. Owing to the various interactions among antioxidants and oxidants, we believe that by measuring the total effects of the oxidants/antioxidants present in an organism, we can obtain more valuable results. Many previous studies have revealed increased oxidative stress in thyroid cancer (Abiaka et al. 2001, Senthil \& Manoharan 2004, Alkhenizan and Hafez 2007, Akinci et al. 2008, Du et al. 2009, Lin et al. 2009, Laatikainen et al. 2010, Lassoued et al. 2010, Erdamar et al. 2010, Liu et al. 2010, Young et al. 2010). Thus, in our study, in addition to the TAS, we measured serum TOS levels and calculated OSI values. One-way ANOVA showed significant differences among study groups in the levels of TAS and TOS. However, no statistically significant differences were found in serum TAS, TOS, and OSI values among carcinoma histological subtypes. The OSI reflects the redox balance between oxidation and antioxidation in a subject in vivo. Our results showed that OSI values were decreased in thyroid disease patients, indicating that in these participants, the redox balance capability was poor and therefore oxidative stress was inevitable. Thus, OSI levels were lower in patients with thyroid cancer than in those with benign thyroid disease, which were in turn lower than those in the healthy subjects. 


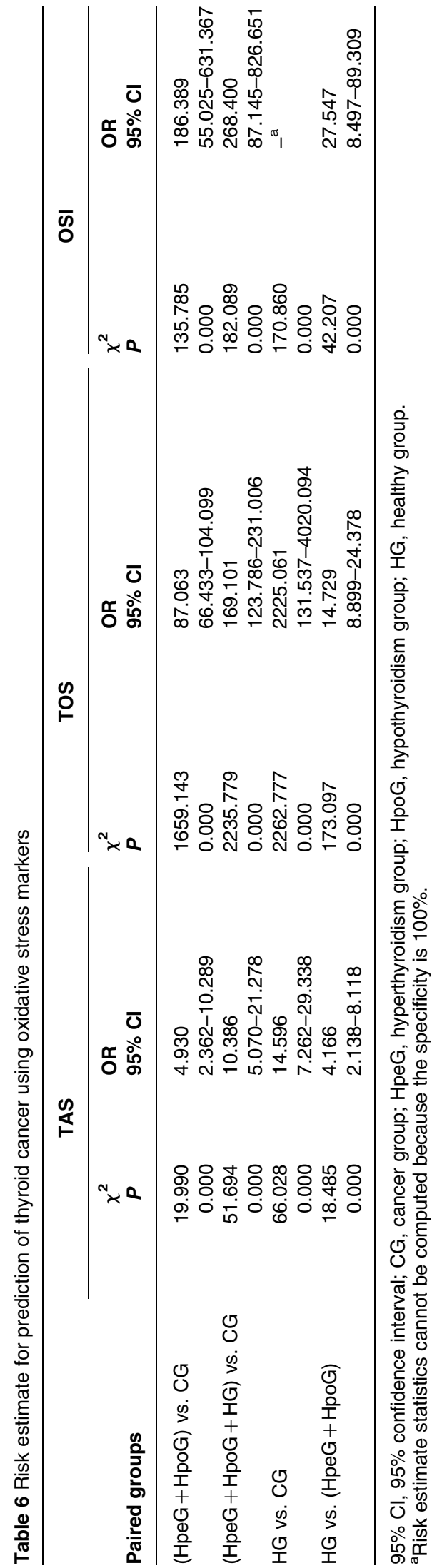

Thyroid hormones are associated with the oxidative and antioxidative status of an organism and thus play an important role in ROS and free radical production (Coria et al. 2009, Erdamar et al. 2010). In the thyroid, ROS and free radicals participate in physiological and pathological processes so that redox imbalance (Senthil \& Manoharan 2004, Akinci et al. 2008, Laatikainen et al. 2010, Young et al. 2010). Antioxidative defense pathways of the organism play a crucial role in reducing the increased levels of free radicals generated by thyroid gland dysfunction (Carmeli et al. 2008). In hyperthyroidism, there is augmented production of ROS because the basal metabolic rate is increased, which in the presence of inadequate antioxidative defense results in the occurrence of oxidative stress in these patients (Saad-Hussein et al. 2011). In hypothyroidism, the basal metabolic rate is decreased and therefore a decrease in free radical production is expected because of the metabolic suppression caused by the lowered thyroid hormone levels (Erdamar et al. 2008). In thyroid cancer, cancerous thyroid tissues have accelerated lipid peroxidation and diminished antioxidant scavenging enzymes, leaving these tissues more vulnerable to the toxic effects of some free radical species (Erdamar et al. 2010). Our study, however, demonstrated no significant correlations between various oxidative stress markers and thyroid profiles using multiple linear regression analysis (Table 3 ). These results suggested that no direct association or not a linear relationship in serum between variations of the levels of thyroid hormones and redox imbalance were caused by thyroid gland dysfunction, although the thyroid hormone can regulate oxidative metabolism and affect enzymatic antioxidants such as SOD catalase, GPx, and glutathione reductase levels and non-enzymatic antioxidants such as vitamin $\mathrm{E}$ and $\mathrm{C}$, glutathione, and uric acid levels in vivo. Possible explanations for this phenomenon include 1) a rigorous and complex antioxidant defense system in the body, 2) the impact on radical chain reactions (accelerated or delayed) by the altered levels of oxidants/antioxidants, 3) the partial effect on oxidant/ antioxidants that are subject to change, or 4) other unknown causes to be investigated.

A previous study confirmed that markers of oxidative stress can be divided into three categories: 1) the formation of modified molecules by free radical reactions, 2) the consumption or induction of antioxidant molecules or enzymes, and 3 ) the activation or inhibition of transcription factors (Erdamar et al. 2010). The category of oxidative stress markers most likely to change when thyroid function is abnormal requires 
further study. However, should we need to explore what kind of antioxidants level is decreased? It is worth considering. To simply understand the oxidative stress status of patients, it is sufficient to measure the TAS and TOS and calculate the OSI. However, concerning the study of oxidative stress for thyroid cancer patients and/or benign thyroid disease patients, most researchers only determined the TAS (Mayer et al. 2004, Bacic-Vrca et al. 2005, Andryskowski \& Owczarek 2007, IshShalom et al. 2008, Torun et al. 2009), some also determined the TOS (Santi et al. 2010, Aslan et al. 2011), and very few studies also calculated the OSI (Aslan et al. 2011) values. However, the TAS had the least accuracy (both AUC and YI are minimal) in our study for the differential diagnosis of thyroid cancer from disease controls and/or healthy controls (Table 4 and Fig. 1). ROC analysis revealed that a perfect test would have an AUC of 1.000 , sensitivity of $100 \%$, a specificity of $100 \%$, and a YI of 1.000 at a cutoff value of 1.01 OSI to distinguish thyroid cancer patients from the healthy population while both AUC and YI are maximal in other statistic groups (Table 4). These results suggest that the OSI may be a good candidate marker for distinguishing thyroid cancer from disease controls and/or healthy controls. Therefore, to obtain a comprehensive understanding of the oxidative stress status of a subject, we should not only measure the TAS and TOS but also calculate the OSI. It can be speculated that TAS is not practiced widely by researchers and clinicians because it reflects the oxidative stress with one-sidedness, i.e. the specificity is lower. Risk estimate statistics have shown that both TOS and OSI were better indicators than TAS either for discriminating thyroid cancer from controls (including disease and healthy controls) or for discriminating thyroid disease from healthy controls (Table 5). This result demonstrated once again that if we want to fully understand the oxidative stress status of patients in vivo, not only TAS and TOS measurements but also the calculation of OSI are necessary.

Recently, one of the focuses on the molecular pathogenesis of thyroid cancer is BRAFV600E mutation. BRAFV600E is a constitutively active onco-kinase and is the most common genetic alteration in PTC and ATC, albeit at a lower frequency (Nucera et al. 2011). BRAFV600E through the mitogenactivated protein kinase signaling pathway may control a network of genes crucial in integrating and regulating the extracellular and intracellular signaling in thyroid cancer cells. The BRAFV600E mutation in some studies has been significantly associated with extra-thyroidal extension, metastases, and recurrence in patients with PTC and ATC (Nucera et al. 2010). Recent investigations have suggested that oxidative stress caused by inflammation may be in part responsible for mutations of the BRAF gene (MartinezCadenas et al. 2011). Other authors also found that oxidative stress plays a critical role in inactivating mutant BRAF by geldanamycin derivatives (Fukuyo et al. 2008). These findings are similar to those of this study. However, the exact mechanism of BRAFV600E mutation induced by oxidative stress needs to explore.

Collecting tissue samples can cause injury, and the pre-analytical specimen treatment is complicated. Furthermore, it is difficult to develop the appropriate reference range, and the results may only reflect the local tissue levels in patients. In contrast, collecting blood samples can overcome these limitations. Therefore, we chose to use blood samples for the current study not only to facilitate scientific research but also to pave the way for future clinical application.

In this study, our observations suggest that the serum TOS and OSI values were higher and TAS levels were lower in patients with thyroid cancer than in controls. There were no correlations between thyroid profiles and oxidative/antioxidative parameters. Therefore, increased levels of oxidants and decreased levels of antioxidants may provide evidence for thyroid patients who were exposed to potent oxidative stress. OSI values may be more useful oxidative stress biomarkers than TAS and TOS for monitoring the clinical status of thyroid cancer. Further investigations are required to clarify the role of oxidative stress in the thyroid carcinogenesis. These results may shed light on the development of novel methods to distinguish thyroid cancer patients from disease controls and/or healthy controls.

\section{Declaration of interest}

The authors declare that there is no conflict of interest that could be perceived as prejudicing the impartiality of the research reported.

\section{Funding}

The work was supported by the People's Republic of China Ministry of Science and Technology (2006AA020905) and BioSino Bio-technology and Science, Inc.

\section{References}

Abiaka C, Al-Awadi F, Al-Sayer H, Gulshan S, Behbehani A, Farghally M \& Simbeye A 2001 Serum antioxidant and cholesterol levels in patients with different types of cancer. Journal of Clinical Laboratory Analysis $\mathbf{1 5}$ 324-330. (doi:10.1002/jcla.1045) 
Akinci M, Kosova F, Cetin B, Sepici A, Altan N, Aslan S \& Cetin A 2008 Oxidant/antioxidant balance in patients with thyroid cancer. Acta Cirúrgica Brasileira 23 551-554. (doi:10.1590/S0102-86502008000600013)

Alkhenizan A \& Hafez K 2007 The role of vitamin E in the prevention of cancer: a meta-analysis of randomized controlled trials. Annals of Saudi Medicine 27 409-414. (doi:10.4103/0256-4947.51459)

Andryskowski G \& Owczarek T 2007 The evaluation of selected oxidative stress parameters in patients with hyperthyroidism. Polskie Archiwum Medycyny Wewnetrznej 117 285-289.

Aslan M, Cosar N, Celik H, Aksoy N, Dulger AC, Begenik H, Soyoral YU, Kucukoglu ME \& Selek S 2011

Evaluation of oxidative status in patients with hyperthyroidism. Endocrine 40 285-289. (doi:10.1007/s12020-0119472-3)

Aycicek A \& Erel O 2007 Total oxidant/antioxidant status in jaundiced newborns before and after phototherapy. Jornal de Pediatria 83 319-322. (doi:10.2223/JPED. 1645)

Aycicek A, Erel O \& Kocyigit A 2005 Decreased total antioxidant capacity and increased oxidative stress in passive smoker infants and their mothers. Pediatrics International 47 635-639. (doi:10.1007/s12020-0119472-3)

Bacic-Vrca V, Skreb F, Cepelak I, Mayer L, Kusic Z \& Petres B 2005 The effect of antioxidant supplementation on superoxide dismutase activity, $\mathrm{Cu}$ and $\mathrm{Zn}$ levels, and total antioxidant status in erythrocytes of patients with Graves' disease. Clinical Chemistry and Laboratory Medicine 43 383-388. (doi:10.1515/CCLM.2005.069)

Boelaert K 2009 The association between serum TSH concentration and thyroid cancer. Endocrine-Related Cancer 16 1065-1072. (doi:10.1677/ERC-09-0150)

Carmeli E, Bachar A, Barchad S, Morad M \& Merrick J 2008 Antioxidant status in the serum of persons with intellectual disability and hypothyroidism: a pilot study. Research in Developmental Disabilities 29 431-438. (doi:10.1016/j.ridd.2007.08.001)

Coria MJ, Pastrán AI \& Gimenez MS 2009 Serum oxidative stress parameters of women with hypothyroidism. Acta Bio-Medica 80 135-139.

Du ZX, Zhang HY, Meng X, Guan Y \& Wang HQ 2009 Role of oxidative stress and intracellular glutathione in the sensitivity to apoptosis induced by proteasome inhibitor in thyroid cancer cells. BMC Cancer 9 56-67. (doi:10.1186/1471-2407-9-56)

Erdamar H, Demirci H, Yaman H, Erbil MK, Yakar T, Sancak B, Elbeg S, Biberoğlu G \& Yetkin I 2008 The effect of hypothyroidism, hyperthyroidism, and their treatment on parameters of oxidative stress and antioxidant status. Clinical Chemistry and Laboratory Medicine 46 1004-1010. (doi:10.1515/CCLM.2008.183)

Erdamar H, Cimen B, Gülcemal H, Saraymen R, Yerer B \& Demirci H 2010 Increased lipid peroxidation and impaired enzymatic antioxidant defense mechanism in thyroid tissue with multinodular goiter and papillary carcinoma. Clinical Biochemistry 43 650-654. (doi:10.1016/j.clinbiochem.2010.02.005)

Erel O 2004 A novel automated direct measurement method for total antioxidant capacity using a new generation, more stable ABTS radical cation. Clinical Biochemistry 37 277-285. (doi:10.1016/j.clinbiochem. 2003.11.015)

Erel O 2005 A new automated colorimetric method for measuring total oxidant status. Clinical Biochemistry 38 1103-1111. (doi:10.1016/j.clinbiochem.2005.08.008)

Fukuyo Y, Inoue M, Nakajima T, Higashikubo R, Horikoshi NT, Hunt C, Usheva A, Freeman ML \& Horikoshi N 2008 Oxidative stress plays a critical role in inactivating mutant BRAF by geldanamycin derivatives. Cancer Research 68 6324-6330. (doi:10.1158/0008-5472.CAN-07-6602)

Harma M, Harma M \& Erel O 2005 Oxidative stress in women with preeclampsia. American Journal of Obstetrics and Gynecology 192 656-657. (doi:10.1016/j. ajog.2004.07.085)

Ish-Shalom S, Durleshter L, Segal E \& Nagler RM 2008 Sialochemical and oxidative analyses in radioactive I131-treated patients with thyroid carcinoma. European Journal of Endocrinology 158 677-681. (doi:10.1530/ EJE-07-0634)

Laatikainen LE, Castellone MD, Hebrant A, Hoste C, Cantisani MC, Laurila JP, Salvatore G, Salerno P, Basolo F, Näsman J et al. 2010 Extracellular superoxide dismutase is a thyroid differentiation marker downregulated in cancer. Endocrine-Related Cancer 17 785-796. (doi:10.1677/ERC-10-0021)

Lassoued S, Mseddi M, Mnif F, Abid M, Guermazi F, Masmoudi H, El Feki A \& Attia H 2010 A comparative study of the oxidative profile in Graves' disease, Hashimoto's thyroiditis, and papillary thyroid cancer. Biological Trace Element Research 138 107-115. (doi:10.1007/s12011-010-8625-1)

Lin JC, Kuo WR, Chiang FY, Hsiao PJ, Lee KW, Wu CW \& Juo SH 2009 Glutathione peroxidase 3 gene polymorphisms and risk of differentiated thyroid cancer. Surgery 145 508-513. (doi:10.1016/j.surg.2008.12.008)

Liu B, Kuang A, Huang R, Zhao Z, Zeng Y, Wang J \& Tian R 2010 Influence of vitamin $\mathrm{C}$ on salivary absorbed dose of $131 \mathrm{I}$ in thyroid cancer patients: a prospective, randomized, single-blind, controlled trial. Journal of Nuclear Medicine 51 618-623. (doi:10.2967/jnumed.109.071449)

Martinez-Cadenas C, Bosch N, Peñas L, Flores-Couce E, Ochoa E, Munárriz J, Aracil JP, Tajahuerce M, Royo R, Lozoya R et al. 2011 Malignant melanoma arising from a perianal fistula and harbouring a $B R A F$ gene mutation: a case report. BMC Cancer 11 343. (doi:10.1186/14712407-11-343)

Mayer L, Romić Z, Skreb F, Bacić-Vrca V, Cepelak I, ZanićGrubisić T \& Kirin M 2004 Antioxidants in patients with hyperthyroidism. Clinical Chemistry and Laboratory Medicine 42 154-158. (doi:10.1515/CCLM.2004.028) 
Nucera C, Lawler J, Hodin R \& Parangi S 2010 The BRAFV600E mutation: what is it really orchestrating in thyroid cancer? Oncotarget 1 751-756. (doi:10.1634/ theoncologist.2010-0317)

Nucera C, Nehs MA, Nagarkatti SS, Sadow PM, Mekel M, Fischer AH, Lin PS, Bollag GE, Lawler J, Hodin RA et al. 2011 Targeting BRAFV600E with PLX4720 displays potent antimigratory and anti-invasive activity in preclinical models of human thyroid cancer. Oncologist 16 296-309.

Saad-Hussein A, Hamdy H, Aziz HM \& Mahdy-Abdallah H 2011 Thyroid functions in paints production workers and the mechanism of oxidative-antioxidants status. Toxicology and Industrial Health 27 257-263. (doi:10. 1177/0748233710386409)

Santi A, Duarte MM, Moresco RN, Menezes C, Bagatini MD, Schetinger MR \& Loro VL 2010 Association between thyroid hormones, lipids and oxidative stress biomarkers in overt hypothyroidism. Clinical Chemistry and Laboratory Medicine 48 1635-1639. (doi:10.1515/ CCLM.2010.309)

Senthil N \& Manoharan S 2004 Lipid peroxidation and antioxidants status in patients with papillary thyroid carcinoma in India. Asia Pacific Journal of Clinical Nutrition 13 391-395.

Torun AN, Kulaksizoglu S, Kulaksizoglu M, Pamuk BO, Isbilen E \& Tutuncu NB 2009 Serum total antioxidant status and lipid peroxidation marker malondialdehyde levels in overt and subclinical hypothyroidism. Clinical Endocrinology 70 469-474. (doi:10.1111/j.1365-2265. 2008.03348.x)

Uttara B, Singh AV, Zamboni P \& Mahajan RT 2009 Oxidative stress and neurodegenerative diseases: a review of upstream and downstream antioxidant therapeutic options. Current Neuropharmacology 7 65-74. (doi:10. 2174/157015909787602823)

Valko M, Leibfritz D, Moncol J, Cronin MT, Mazur M \& Telser J 2007 Free radicals and antioxidants in normal physiological functions and human disease. International Journal of Biochemistry \& Cell Biology 39 44-84. (doi:10.1016/j.biocel.2006.07.001)

Wells PG, McCallum GP, Chen CS, Henderson JT, Lee CJ, Perstin J, Preston TJ, Wiley MJ \& Wong AW 2009 Oxidative stress in developmental origins of disease: teratogenesis, neurodevelopmental deficits, and cancer. Toxicological Sciences 108 4-18. (doi:10.1093/toxsci/kfn263)

Young O, Crotty T, O'Connell R, O’Sullivan J \& Curran AJ 2010 Levels of oxidative damage and lipid peroxidation in thyroid neoplasia. Head \& Neck 32 750-756. (doi:10. 1002/hed.21247)

Received in final form 5 October 2011

Accepted 14 October 2011

Made available online as an Accepted Preprint 14 October 2011 